\title{
CARACTERÍSTICAS DOS PACIENTES ATENDIDOS PELA FISIOTERAPIA EM UM HOSPITAL PÚBLICO
}

Janaina Andrade dos Santos, Caroline Braun, Guilherme Henrique Dalaqua Grande, Rafael Zambelli Pinto, Susimary Aparecida Trevisan Padulla.

Universidade Estadual Paulista - UNESP, Curso de Fisioterapia, Departamento de Fisioterapia Presidente Prudente, SP. E-mail: jane andrade06@hotmail.com

\section{RESUMO}

No ambiente hospitalar, a assistência promovida pelos profissionais da saúde coopera para recuperar a condição clínica dos pacientes para retornar à realidade com qualidade de vida. Objetivo descrever o perfil dos pacientes do SUS atendidos pelo serviço de fisioterapia de uma universidade nos anos de 2012 e 2013, em um hospital público de Presidente Prudente a fim de conhecer os usuários e planejar o processo de assistência em saúde. Participaram 1048 pacientes em 2012 e 1101 em 2013 encaminhados para a fisioterapia que internaram no hospital pelo SUS. Resultados houve uma prevalência de idosos, e do sexo masculino, com destaque para as especialidades oncologia (26,6\%) em 2012 e para cardiologia (31,3\%) em 2013. O tempo médio de internação foi maior em 2012 com 11,1 $\pm 10,8$ dias. Concluímos discussões são necessárias para adequar a formação do profissional fisioterapeuta na atuação hospitalar permitindo direcionamento do tratamento prestado e melhora no planejamento do processo de assistência à saúde.

Palavras-chave: Fisioterapia; Hospital; Caracterização.

\section{CHARACTERISTICS OF PATIENTS TREATED BY PHYSICAL THERAPY IN A PUBLIC HOSPITAL.}

\begin{abstract}
In the hospital environment, promoted by health professionals cooperates assistance to recover the clinical condition of the patients to return to reality with quality of life. Objective to describe the profile of UHS patients treated by physiotherapy service at a university in the years 2012 and 2013, in a Presidente Prudente public hospital in order to meet users and plan the process of health care. Participants 1048 patients in 2012 and 1101 in 2013 referred for physical therapy who were hospitalized at the hospital by SUS. Results there was a prevalence of elderly and male, with an emphasis on oncology specialties (26.6\%) in 2012 and for cardiology (31.3\%) in 2013. The average length of hospitalization was higher in 2012 with $11,1 \pm 10.8$ days. We concluded discussions are necessary to adapt the education of physiotherapist in hospital performance allowing orientation of service treatment and improvement in the design of the health care process.
\end{abstract}

Keywords: physiotherapy; hospital; characterization. 


\section{INTRODUÇÃO}

A procura as redes públicas de saúde de média e alta complexidade como hospitais, se dá pelos indivíduos com o objetivo de diagnóstico para as doenças agudas ou crônicas, acompanhamento, internação, exames e cirurgias visando à promoção da saúde ou na maioria das vezes o tratamento da doença ${ }^{1,2}$. Em 2005 no Brasil foram registrados uma média de 6,96 internações hospitalares por 100 habitantes no Sistema Único de Saúde (SUS),o que gerou um gasto no sistema de $\mathrm{R} \$ \mathbf{6 , 9 6}$ bilhões neste ano. A região Sudeste foi a que apresentou menor taxa de internação hospitalar no SUS, com valor médio em 2005 de 5,6. ${ }^{3}$

No ambiente hospitalar, a assistência promovida pelos profissionais da saúde coopera para recuperar a condição clínica dos pacientes, a fim de que eles possam retornar à realidade em que se inserem com qualidade de vida. No entanto, a hospitalização pode submeter o individuo aos efeitos da imobilidade, como o descondicionamento físico, fraqueza muscular,alterações pulmonares, tegumentar, gastrointestinais, depressão, queda no nível de participação em atividades de autocuidados, entre outros, o que se associa à maiores índices de complicações e a reabilitação prolongada ${ }^{4,5}$.

Diante do quadro de disfunções associada ao imobilismo a fisioterapia tem sido amplamente requisitada, a fim de melhorar a capacidade funcional do paciente e restaurar a sua independência física, diminuindo os riscos do repouso e o tempo de permanência hospitalar ${ }^{5,6}$.

No âmbito respiratório a fisioterapia utiliza meios e técnicas de avaliação e tratamento que, contribui para prevenir, reverter ou minimizar disfunções ventilatórias, promovendo a máxima funcionalidade e qualidade de vida dos pacientes ${ }^{8}$; por melhorara função respiratória, mantendo níveis adequados de oxigenação e de gás carbônico na circulação, preservando a função pulmonar e a manutenção da expansibilidade da caixa torácica ${ }^{9}$. Cinesioterapia, cicloergômetro, mobilização passiva, sentar na borda da cama, ortostatismo, e deambulação são exemplos de desfechos simples e efetivos executados na mobilização precoce ${ }^{10,11}$.

A importância desse conhecimento está relacionada ao direcionamento do tratamento prestado, a formação teórica seja ela na graduação ou para a prática clínica, além de possibilitar a criação de instrumentos de avaliação e orientação para o sucesso de pesquisas clínicas. Objetivo deste estudo foi descrever o perfil dos pacientes do SUS atendidos pelo serviço de fisioterapia de uma universidade nos anos de 2012 e 2013, em um hospital público de Presidente Prudente a fim de conhecer os usuários e planejar o processo de assistência em saúde. 


\section{METODOLOGIA}

Este é um estudo observacional, tipo retrospectivo, de caráter descritivo realizado em um hospital público da cidade de Presidente Prudente/SP, com pacientes atendidos no SUS e que realizaram fisioterapia. O estudo foi desenvolvido de modo a garantir o cumprimento dos preceitos da Resolução 196/96, tendo sido aprovado pelo Comitê de Ética da Santa Casa e pelo Comitê de Ética em Pesquisa da Faculdade de Ciências e Tecnologia- FCT/Unesp (Proc. $n^{\circ}$ 02592612.8.0000.5402) obedecendo a Resolução 466/2012 do Conselho Nacional de Saúde de $12 / 12 / 2012$.

Seus atendimentos são tanto a usuários do sistema público de saúde, quanto do sistema privado. Do total de 11 unidades de internação que possui, foram utilizados dados dos pacientes internados em: duas clínicas de internações gerais para adultos do SUS com 30 leitos cada; uma clínica de internações pós-cirúrgicas para adultos do SUS com 18 leitos; uma unidade de pediatria com 14 leitos; e uma unidade de terapia intensiva coronariana com 8 leitos.

O serviço de fisioterapia é realizado por uma parceria do hospital público e do Serviço Integrado de Fisioterapia com o departamento do curso de fisioterapia da Faculdade de Ciências e Tecnologia da Universidade Estadual Paulista - Fct/Unesp.

Foram incluídos no estudo todos os pacientes atendidos pelo serviço de fisioterapia durante os anos de 2012 e 2013. Pacientes com prontuários de avaliação incompletos ou ilegíveis, o que impossibilitava a obtenção dos dados, foram excluídos do estudo.

Os dados de cada paciente foram extraídos, por meio de um formulário próprio, dos prontuários de avaliação fisioterapêutica preenchidas nos anos de 2012 e 2013 . Foram coletados dados demográficos como sexo e idade, e dados clínicos, como a hipótese diagnóstica de internação, a especialidade médica, a data de admissão, a data de alta e o tempo de permanência hospitalar.

Análise dos dados foi construído um banco de dados, em planilhas do programa Microsoft Office Excel 2007, os quais foram analisados de forma descritiva. Análise dos anos 2012 e 2013, foram realizadas separadamente para cada ano. Dados dicotômicos e categóricos, dados com distribuição normal e dados com distribuição não-normal, foram reportados utilizando frequência (proporção), média (desvio padrão) e mediana (intervalo interquartílico), respectivamente. Foi utilizado o software estatístico IBM SPSS versão 20.0 (IBM corporation, Somers, NY, USA).

\section{Resultados}

Segundo dados das fichas de registro, 1048 pacientes foram atendidos pela fisioterapia em 2012 e 1101 foram atendidos no ano de 2013. Houve uma perda amostral de dados de 10 (1\%) 
pacientes em 2012 e de 24 (2\%) em 2013, totalizando 34 (2\%) pacientes que não participaram da análise por apresentar dados incompletos no prontuário.

A média de idade foi similar durante os dois anos, com predomínio do sexo masculino, o que pode ser observado na tabela 1 . Mais de $60 \%$ dos atendidos foram considerados idosos, com faixa etária entre 60 e 74 anos, (60,8\% em 2012 e 61,7\% em 2013), sendo a média de idade de $60,2 \pm 20$ e 61,2 $\pm 19,4$ respectivamente. A tabela 1 ainda mostra a especialidade médica em que os pacientes se enquadraram a partir do diagnóstico. As maiores prevalências em porcentagem foram para as especialidades Cardiologia e Oncologia em ambos os períodos, destacando que em 2013, 31,3\% dos atendimentos foram para as doenças do coração, contra 18,2\% em 2012.

Tabela1. Prevalência de idade, sexo e especialidades médicas nos anos de 2012 e 2013.

\begin{tabular}{ccc}
\hline & $\mathbf{2 0 1 2}$ & $\mathbf{2 0 1 3}$ \\
\hline $\begin{array}{c}\text { idade, média (DP) } \\
\text { idade, } \mathbf{n}(\%)\end{array}$ & $60,2(20)$ & $61,2(19,4)$ \\
$\mathbf{1 8}$ & $54(5,2)$ & $55(5,0)$ \\
$\mathbf{1 9 - 3 9}$ & $88(8,4)$ & $79(7,2)$ \\
$\mathbf{4 0 - 5 9}$ & $277(26,4)$ & $288(26,2)$ \\
$\mathbf{6 0 - 7 4}$ & $379(36,2)$ & $380(34,5)$ \\
$>\mathbf{7 5}$ & $250(23,9)$ & $299(27,2)$ \\
Sexo & & \\
Feminino & $467(45)$ & $489(44)$ \\
Masculino & $581(55)$ & $612(56)$ \\
& & \\
Especialidades & & $88(8,0)$ \\
Neurologia & $97(9,3)$ & $129(11,7)$ \\
Pneumologia & $110(10,5)$ & $345(31,3)$ \\
Cardiologia & $191(18,2)$ & $70(6,4)$ \\
Gastroenterologia & $98(9,4)$ & $50(4,5)$ \\
Nefrologia & $62(5,9)$ & $243(22,1)$ \\
Oncologia & $279(26,6)$ & $127(11,5)$ \\
Ortopedia & $141(13,5)$ & $10(0,9)$ \\
Hematologia & $20(1,9)$ & $39(3,5)$ \\
Pediatria & $47(4,5)$ & \\
& & \\
& & \\
\hline
\end{tabular}


O tempo médio de internação em dias em 2012 que um paciente deste estudo ficou hospitalizado foi de $11,1 \pm 10,8$. Para 2013 , esse tempo corresponde a $9,3 \pm 6,8$ dias.

A Tabela 2 mostra o tempo médio de internação e a prevalência do sexo e da idade de acordo com cada especialidade para os anos de 2012 e 2013. Exceto na Pediatria, a idade por especialidade não difere muito da média encontrada por ano, tanto para 2012 como para 2013. Os pacientes internados na pediatria apresentam a menor média de dias de internação, seguido pela especialidade cardiologia, com 9,1 $\pm 7,0$ dias em 2012 e pneumologia, com 8,1 $\pm 7,9$, em 2013. As especialidades que apresentaram maiores médias em dias de internação foram neurologia, gastroenterologia e nefrologia em 2012, com média de 13,3 dias e em 2013, oncologia com 10,3 dias.

Tabela 2. Idade, sexo e tempo de internação de acordo com especialidades médicas.

\begin{tabular}{|c|l|l|l|l|l|l|}
\hline & \multicolumn{3}{|c|}{$\mathbf{2 0 1 2}$} & \multicolumn{3}{c|}{$\mathbf{2 0 1 3}$} \\
\hline & idade & $\begin{array}{l}\text { dias de } \\
\text { internação }\end{array}$ & $\begin{array}{l}\text { Sexo } \\
\text { feminino }\end{array}$ & idade & $\begin{array}{l}\text { dias de } \\
\text { internação }\end{array}$ & $\begin{array}{l}\text { Sexo } \\
\text { feminino }\end{array}$ \\
\hline Especialidades & & & & & & \\
\hline Neurologia & $64,1(17,0)$ & $13,3(17,3)$ & $49(51 \%)$ & $64,1(21,2)$ & $9,6(9,2)$ & $35(40 \%)$ \\
\hline Pneumologia & $68,2(17,9)$ & $9,9(8,6)$ & $40(36 \%)$ & $67,8(17,0)$ & $8,1(7,9)$ & $64(49 \%)$ \\
\hline Cardiologia & $65,5(14,0)$ & $9,1(7,0)$ & $91(47 \%)$ & $65,0(13,3)$ & $9,5(5,7)$ & $151(44 \%)$ \\
\hline Gastroenterologia & $61,7(17,4)$ & $13,3(10,3)$ & $52(53 \%)$ & $56,7(21,0)$ & $8,7(6,0)$ & $35(50 \%)$ \\
\hline Nefrologia & $62,7(13,1)$ & $13,3(13,0)$ & $30(48 \%)$ & $63,4(14,5)$ & $9,6(5,4)$ & $23(46 \%)$ \\
\hline Oncologia & $60,8(13,6)$ & $12,6(9,5)$ & $113(41 \%)$ & $61,5(13,3)$ & $10,3(6,8)$ & $99(41 \%)$ \\
\hline Ortopedia & $58,4(19,7)$ & $10,7(12,4)$ & $63(45 \%)$ & $60,0(20,0)$ & $9,0(7,9)$ & $68(53 \%)$ \\
\hline Hematologia & $67,4(17,9)$ & $11,5(7,6)$ & $6(30 \%)$ & $60,5(22,7)$ & $10,1(5,2)$ & $01(10 \%)$ \\
\hline Pediatria & $4,0(4,2)$ & $7,6(9,0)$ & $23(49 \%)$ & $5,3(5,2)$ & $6,2(3,0)$ & $13(33 \%)$ \\
\hline
\end{tabular}

O gráfico 1 apresenta as internações em 2012 e 2013, por especialidade, de acordo com o período do ano que ocorreram. Observa-se o predomínio por atendimentos na área de oncologia em 2012, e por atendimentos em cardiologia no ano de 2013.

Gráfico 1. Distribuição por meses do ano das especialidades atendidas em 2012 e 2013.
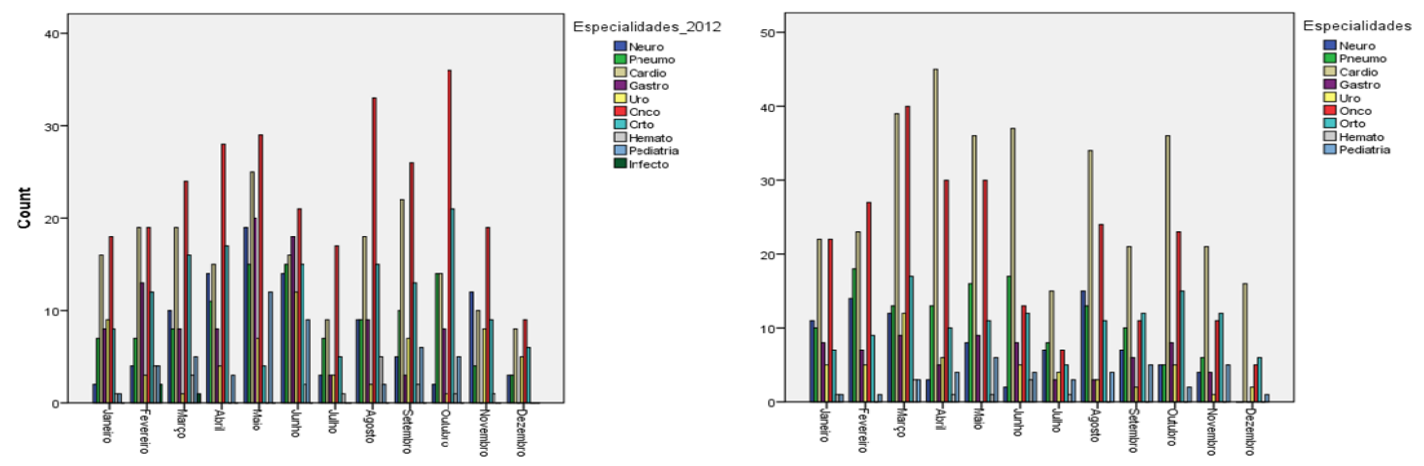


\section{DISCUSSÃO}

A partir das análises dos pacientes encaminhados para a fisioterapia do hospital público deste estudo observou-se o predomínio de pacientes idosos do sexo masculino. Em 2012 os atendimentos prestados foram maiores na área de oncologia (26,6\%) e em 2013 na área de cardiologia (31,3\%). O tempo médio de internação foi maior em 2012 com 11,1 $\pm 10,8$ dias.

Os estudos em hospitais generalistas ${ }^{12,13}$ no Brasil apontam alta prevalência de idosos e homens em suas internações, que correspondem ao achados deste estudo. A população idosa utiliza os serviços hospitalares de maneira mais intensiva que os demais grupos etários, implicando maiores custos, duração do tratamento e recuperação mais lenta ${ }^{13,14}$. Um estudo realizado na região sul do Brasil apontou que pessoas com sessenta anos ou mais têm uma chance de internação 4,96 vezes maior que a faixa etária de 14-19 anos ${ }^{15}$.

A outra especialidade prevalente foi a oncologia. De acordo com recentes pesquisas do Ministério da Saúde, o câncer se tornará um problema maior nas próximas décadas, sendo o número de mortes por câncer no mundo estimadas para chegar a mais de 11 milhões em $2030^{16}$.

Este estudo aponta alta prevalência dos tratamentos fisioterapêuticos em pacientes com algum tipo de câncer. Esses e outros achados podem levar a discussões quanto à formação curricular e preparação para a prática dos alunos e futuros profissionais, por se tratar de uma especialidade não muito abordada na graduação, porém muito crescente no ambiente hospitalar. Estudos futuros seriam importantes com o objetivo de evidenciar as técnicas fisioterapêuticas, ou até mesmo buscar as técnicas mais utilizadas e eficientes de acordo com as especialidades, embasando a fisioterapia no ambiente hospitalar.

\section{CONCLUSÃO}

Portanto conclui-se que os pacientes atendidos pela fisioterapia são em maioria idosos do sexo masculino, com diagnósticos nas áreas de oncologia e cardiologia, e que discussões são necessárias para adequar a formação do profissional fisioterapeuta na atuação hospitalar permitindo direcionamento do tratamento prestado e melhora no planejamento do processo de assistência à saúde.

\section{REFERÊNCIAS}

1. Mendes MFM, Freese E, Guimarães MJB. Núcleo de epidemiologia em hospitais de alta complexidade da rede pública de saúde situados no Recife, Pernambuco: avaliação da implantação. Rev Bras Saúde Matern Infant. 2004; 4(4): 435-47. http://dx.doi.org/10.1590/s1519$\underline{38292004000400013}$ 
2. Conselho Nacional de Secretários de Saúde. Assistência de média e alta complexidade no Sus. $9^{\text {nd }}$ ed. Brasília Copyright, 2007.

3.DATASUS[internet]. Brasília(DF): Ministério da Saúde; 2005. [Citado em 28 de janeiro de 2015]. Disponível em: http://datasus.gov.br

4. Valentin A. The importance of risk reduction in critically ill patients. Curr Opin Crit Care. 2010; 16(5): 482-6. http://dx.doi.org/10.1097/MCC.0b013e32833cb861

5. Barros GF. Treinamento muscular respiratório na revascularização do miocárdio. Rev Bras Cir Cardiovasc. 2010; 25(4): 483-90. http://dx.doi.org/10.1590/S0102-76382010000400011

6. Silva APP, Maynard K, Cruz MR. Efeitos da fisioterapia motora em pacientes críticos: revisão de literatura. Rev Bras Ter Intensiva. 2010; 22(1): 85-91. http://dx.doi.org/10.1590/S0103$\underline{507 \times 2010000100014}$

8. Stiller K. Physiotherapy in intensive care. Towards na evidence basead practice. Chest 2000; 118:1801-13. http://dx.doi.org/10.1378/chest.118.6.1801

9. Yokota $\mathrm{CO}$ et al. Fisioterapia respiratória em pacientes sob ventilação mecânica. Rev Ciência Médica. 2006;15(4):339-45.

10. Pinheiro AR, Christofoletti G. Fisioterapia motora em Pacientes internados na unidade de terapia intensiva: Uma Revisão Sistemática. Rev Bras Ter Intensiva. 2012; 24(2): 188-96. http://dx.doi.org/10.1590/S0103-507X2012000200016

11. Dantas MC, Silva PFSS, Siqueira FHT. Influência da mobilização precoce na força muscular periférica e respiratória em pacientes críticos. Rev Bras Ter Intensiva. 2012; 24(2): 173-8. http://dx.doi.org/10.1590/S0103-507X2012000200013

12. Bento MCC, Cardoso FEF, Torquato JA. Universidade e a formação em saúde no setor hospitalar. Rev Saúde. 2013; 7(3/4): 40-8.

13. Berenstein CK, Wajnman S. Efeitos da estrutura etária nos gastos com internação no Sistema Único de Saúde: uma análise de decomposição para duas áreas metropolitanas brasileiras. Cad Saúde Pública. 2008; 24(10): 2301-13. http://dx.doi.org/10.1590/S0102-311X2008001000011

14. Vieira MS. Perfil geográfico e clínico de pacientes admitidos na UTI através da Central de Regulação de Internações Hospitalares. Com. Ciências Saúde. 2011; 22(3):201-10.

15. Garbinato LR et al. Prevalência de internação hospitalar e fatores associados: um estudo de base populacional em um centro urbano no Sul do Brasil. Cad Saúde Pública. 2007; 23(1): 217-24. http://dx.doi.org/10.1590/S0102-311X2007000100023

16. World Health Organization (WHO). Câncer 2008 [boletim]. Disponível em: http://www.who.int/mediacentre/factsheets/fs297/en/ .Acesso em 20 de janeiro de 2015. 